\title{
A Note on the Regularity Criterion of Weak Solutions of Navier-Stokes Equations in Lorentz Space
}

\author{
Xunwu Yin \\ School of Science, Tianjin Polytechnic University, Tianjin 300387, China \\ Correspondence should be addressed to Xunwu Yin, yinxunwu@hotmail.com \\ Received 3 July 2012; Accepted 7 August 2012 \\ Academic Editor: Yonghong Yao \\ Copyright (C) 2012 Xunwu Yin. This is an open access article distributed under the Creative \\ Commons Attribution License, which permits unrestricted use, distribution, and reproduction in \\ any medium, provided the original work is properly cited. \\ This paper is concerned with the regularity of Leray weak solutions to the 3D Navier-Stokes \\ equations in Lorentz space. It is proved that the weak solution is regular if the horizontal velocity \\ denoted by $\tilde{u}=\left(u_{1}, u_{2}, 0\right)$ satisfies $\tilde{u}(x, t) \in L_{q}\left(0, T ; L_{p, \infty}\left(\mathbf{R}^{3}\right)\right)$ for $2 / q+3 / p=1,3<p<\infty$. The \\ result is obvious and improved that of Dong and Chen (2008) on the Lebesgue space.
}

\section{Introduction and Main Results}

In this note, we consider the regularity criterion of weak solutions of the Navier-Stokes equations in the whole space $\mathbf{R}^{3}$

$$
\begin{gathered}
\partial_{t} u+(u \cdot \nabla) u+\nabla \pi=\Delta u, \\
\nabla \cdot u=0, \\
u(x, 0)=u_{0} .
\end{gathered}
$$

Here $u=\left(u_{1}, u_{2}, u_{3}\right)$ and $\pi$ denote the unknown velocity field and the unknown scalar pressure field. $u_{0}$ is a given initial velocity. For simplicity, we assume that the external force is zero, but it is easy to extend our results to the nonzero external force case. Here and in what follows, we use the notations for vector functions $u, v$,

$$
(u \cdot \nabla) v=\sum_{i=1}^{3} u_{i} \partial_{i} v_{k} \quad(k=1,2,3), \quad \nabla \cdot u=\sum_{i=1}^{3} \partial_{i} u_{i}
$$


For a given initial data $u_{0} \in L^{2}\left(\mathbf{R}^{3}\right)$, Leary in the pioneer work, [1] constructed a global weak solution

$$
u \in L^{\infty}\left(0, \infty ; L^{2}\left(\mathbf{R}^{3}\right)\right) \cap L^{2}\left(0, T ; H^{1}\left(\mathbf{R}^{3}\right)\right)
$$

From that time on, although much effort has been made on the uniqueness and regularity of weak solutions, the question of global regularity or finite time singularity for weak solutions in $\mathbf{R}^{3}$ is still open. One important observation is that the regularity can be derived when certain growth conditions are satisfied. This is known as a regularity criterion problem. The investigation of the regularity criterion on the weak solution stems from the celebrated work of Serrin [2]. Namely, Serrin's regularity criterion can be described as follows. A weak solution $u$ of Navier-Stokes equations is regular if the growth condition on velocity field $u$

$$
u \in L_{p}\left(0, T ; L_{q}\left(\mathbf{R}^{3}\right)\right) \equiv L_{p} L_{q}, \quad \text { for } \frac{2}{p}+\frac{3}{q} \leq 1, \quad 3<q \leq \infty,
$$

holds true.

It should be mentioned that the Serrin's condition (1.4) is important from the point of view of the relation between scaling invariance and regularity criteria of weak solutions; indeed, if a pair $(u, p)$ solves $(1.1)$, then so does $\left(u_{\curlywedge}, p_{\curlywedge}\right)$ defined by

$$
u_{\lambda}(x, t)=\lambda u\left(\lambda x, \lambda^{2} t\right), \quad p_{\lambda}(x, t)=\lambda^{2} p\left(\lambda x, \lambda^{2} t\right)
$$

Scaling invariance means that

$$
\|u\|_{L^{p}\left(0, T ; L^{q}\left(\mathbf{R}^{3}\right)\right)}=\left\|u_{\lambda}\right\|_{L^{p}\left(0, T ; L^{q}\left(\mathbf{R}^{3}\right)\right)}
$$

holds for all $\lambda>0$ and this happens if and only if $p$ and $q$ satisfy (1.4).

Actually, the condition described by (1.4) which involves all components of the velocity vector field $u=\left(u_{1}, u_{2}, u_{3}\right)$ is known as degree -1 growth condition (see Chen and Xin [3] for details), since

$$
\left\|u\left(\lambda \cdot, \lambda^{2} \cdot\right)\right\|_{L_{p} L_{q}}=\|u\|_{L_{p}\left(0, \lambda^{2} T ; L_{q}\left(\mathbf{R}^{3}\right)\right)} \lambda^{-2 / p-3 / q}=\|u\|_{L_{p}\left(0, \lambda^{2} T ; L_{q}\left(\mathbf{R}^{3}\right)\right)} \lambda^{-1} .
$$

The degree -1 growth condition is critical due to the scaling invariance property. That is, $(u(x, t), p(x, t))$ solves (1.1) if and only if $\left(u_{\lambda}(x, t), p_{\lambda}(x, t)\right)$ is a solution of (1.1).

Moreover, this pioneer result [2] has been extended by many authors in terms of velocity $u(x, t)$, the gradient of velocity $\nabla u(x, t)$ or vorticity $w(x, t)=\left(w_{1}, w_{2}, w_{3}\right)=\nabla \times u$ in Lebesgue spaces or Besov spaces, respectively (refer to [4-7] and reference therein).

Actually, the weak solution remains regular when a part of the velocity components or vorticity is involved in a growth condition. On one hand, regularity of the weak solution was recently obtained by Dong and Chen [8] when two velocity components denoted by

$$
\tilde{u}=\left(u_{1}, u_{2}, 0\right)
$$


satisfy the critical growth condition

$$
\tilde{u} \in L_{p} L_{q}, \quad \text { for } \frac{2}{p}+\frac{3}{q}=1, \quad 3<q \leq \infty
$$

It should be mentioned that the weak solution remains regular if the single velocity component satisfies the higher (subcritical) growth conditions (see Zhou [9], Penel and Pokorný [10], Kukavica and Ziane [11], and Cao and Titi [12]). One may also refer to some interesting regularity criteria [13-15] for weak solutions of micropolar fluid flows. It seems difficult to show regularity of weak solutions by imposing Serrin's growth condition on only one component of velocity field for both Navier-Stokes equations and micropolar fluid flows.

However, whether or not the result (1.9) can be improved to the critical weak $L_{p}$ spaces is an interesting and challenging problem, that is to say, when the weak critical growth condition is imposed to only two velocity components. The main difficulty lies in the lack of a priori estimates on two-velocity components $\widetilde{u}$ due to the special structure of the nonlinear convection term in monument equations.

The aim of the present paper is to improve the two-component regularity criterion (1.9) from Lebesgue space to the critical Lorentz space (see the definitions in Section 2) which satisfies the scaling invariance property.

Before stating the main results, we firstly recall the definition of the Leray weak solutions.

Definition 1.1 (Temam, [16]). Let $u_{0} \in L_{2}\left(\mathbf{R}^{3}\right)$ and $\nabla \cdot u_{0}=0$. A vector field $u(x, t)$ is termed as a Leray weak solution of $(1.1)$ on $(0, T)$ if $u$ satisfies the following properties:

(i) $u \in L_{\infty}\left(0, T ; L_{2}\left(\mathbf{R}^{3}\right)\right) \cap L_{2}\left(0, T ; H^{1}\left(\mathbf{R}^{3}\right)\right)$;

(ii) $\partial_{t} u+(u \cdot \nabla) u+\nabla \pi=\Delta u$ in the distribution space $\Phi^{\prime}\left((0, T) \times \mathbf{R}^{3}\right)$;

(iii) $\nabla \cdot u=0$ in the distribution space $\Phi^{\prime}\left((0, T) \times \mathbf{R}^{3}\right)$;

(iv) $u$ satisfies the energy inequality

$$
\|u(t)\|_{L_{2}}^{2}+2 \int_{0}^{t} \int_{\mathbf{R}^{3}}|\nabla u(x, s)|^{2} d x d s \leq\left\|u_{0}\right\|_{L_{2}}^{2}, \quad \text { for } 0 \leq t \leq T
$$

The main results now read as follows.

Theorem 1.2. Suppose $T>0, u_{0} \in H^{1}\left(\mathbf{R}^{3}\right)$ and $\nabla \cdot u_{0}=0$ in the sense of distributions. Assume that $u$ is a Leray weak solution of the Navier-Stokes equations (1.1) in $(0, T)$. If the horizontal velocity denoted by $\tilde{u}=\left(u_{1}, u_{2}, 0\right)$ satisfies the following growth condition:

$$
\int_{0}^{T}\|\tilde{u}(t)\|_{L_{p, \infty}}^{q} d t<\infty, \quad \text { for } \frac{2}{q}+\frac{3}{p}=1, \quad 3<p<\infty,
$$

then $u$ is a regular solution on $(0, T]$.

Remark 1.3. It is easy to verify that the spaces (1.11) satisfy the degree -1 growth conditions due to the scaling invariance property. Moreover, since the embedding relation $L_{p} \hookrightarrow L_{p, \infty}$, Theorem 1.2 is an important improvement of (1.9). 
Remark 1.4. Unlike the previous investigations via two components of vorticity (see $[17,18]$ ) in weak space, of which the approaches are mainly based on the vorticity equations and seem not available in our case here due to the special structure of convection term, the present examination is directly based on the momentum equations. In order to make use of the structure of the nonlinear convection term $(u \cdot \nabla) u$, we study every component of $((u \cdot \nabla) u, \Delta u)$ and estimate them one by one with the aid of the identities $\nabla \cdot u=0$.

\section{Preliminaries and A Priori Estimates}

To start with, let us introduce the definitions of some functional spaces. $L_{p}\left(\mathbf{R}^{3}\right), W^{k, p}\left(\mathbf{R}^{3}\right)$ with $k \in \mathbf{R}, 1 \leq p \leq \infty$ are usual Lebesgue space and Sobolev space.

To define the Lorenz space $L_{p, q}\left(\mathbf{R}^{3}\right)$ with $1 \leq p, q \leq \infty$, we consider a measurable function $f$ and define for $t \geq 0$ the Lebesgue measure

$$
m(f, t):=m\left\{x \in \mathbf{R}^{3}:|f(x)|>t\right\}
$$

of the set $\left\{x \in \mathbf{R}^{3}:|f(x)|>t\right\}$.

Then $f \in L_{p, q}\left(\mathbf{R}^{3}\right)$ if and only if

$$
\begin{gathered}
\|f\|_{L_{p, q}}=\left(\int_{0}^{\infty} t^{q}(m(f, t))^{q / p} \frac{d t}{t}\right)^{1 / q}<\infty \quad \text { for } 1 \leq q<\infty, \\
\|f\|_{L_{p, \infty}}=\sup _{t \geq 0}\left(t(m(f, t))^{1 / p}\right)<\infty \quad \text { for } q=\infty .
\end{gathered}
$$

Actually, Lorentz space $L_{p, q}\left(\mathbf{R}^{3}\right)$ may be alternatively defined by real interpolation (see Bergh and Löfström [19] and Triebel [20])

$$
L_{p, q}\left(\mathbf{R}^{3}\right)=\left(L_{p_{1}}\left(\mathbf{R}^{3}\right), L_{p_{2}}\left(\mathbf{R}^{3}\right)\right)_{\theta, q^{\prime}}
$$

with

$$
\frac{1}{p}=\frac{1-\theta}{p_{1}}+\frac{\theta}{p_{2}}, \quad 1 \leq p_{1}<p<p_{2} \leq \infty .
$$

Especially, $\|f\|_{L_{q, \infty}}$ is equivalent to the norm

$$
\sup _{0<|E|<\infty}|E|^{1 / q-1} \int_{E}|f(x)| d x
$$

and thus it readily seen that

$$
L_{p}\left(\mathbf{R}^{3}\right)=L_{p, p}\left(\mathbf{R}^{3}\right) \subset L_{p, q}\left(\mathbf{R}^{3}\right) \subset L_{p, \infty}\left(\mathbf{R}^{3}\right), \quad 1<p<q<\infty .
$$


In order to prove Theorem 1.2, it is sufficient to examine a priori estimates for smooth solutions of (1.1) described in the following.

Theorem 2.1. Let $T>0, u_{0} \in H^{1}\left(\mathbf{R}^{3}\right)$ with $\nabla \cdot u_{0}=0$. Assume that $u(x, t)$ is a smooth solution of (1.1) on $\mathbf{R}^{3} \times(0, T)$ and satisfies the growth conditions (1.11). Then

$$
\sup _{0<t<T}\|\nabla u(t)\|_{L_{2}}^{2}+\int_{0}^{T}\|\Delta u(t)\|_{L_{2}}^{2} d t \leq c\left\|\nabla u_{0}\right\|_{L_{2}}^{2} \exp \left\{\int_{0}^{T}\|\tilde{u}(t)\|_{L_{p, \infty}}^{q} d t\right\}
$$

holds true.

Proof of Theorem 2.1. Taking inner product of the momentum equations of (1.1) with $\Delta u$ and integrating by parts, one shows that

$$
\frac{1}{2} \frac{d}{d t}\|\nabla u(t)\|_{L_{2}}^{2}+\|\Delta u(t)\|_{L_{2}}^{2} \leq-\sum_{i, j, k=1}^{3} \int_{\mathbf{R}^{3}} u_{i} \partial_{i} u_{j} \partial_{k k} u_{j} d x
$$

In order to estimate the right-hand side of (2.8), with the aid of the divergence-free condition $\sum_{i=1}^{3} \partial_{i} u_{i}=0$ and integration by parts, observe that

$$
\begin{aligned}
-\sum_{i, j, k=1}^{3} \int_{\mathbf{R}^{3}} u_{i} \partial_{i} u_{j} \partial_{k k} u_{j} d x= & \sum_{i, j, k=1}^{3} \int_{\mathbf{R}^{3}} \partial_{k}\left(u_{i} \partial_{i} u_{j}\right) \partial_{k} u_{j} d x \\
= & \sum_{i, j, k=1}^{3} \int_{\mathbf{R}^{3}} \partial_{k} u_{i} \partial_{i} u_{j} \partial_{k} u_{j} d x+\frac{1}{2} \sum_{i, j, k=1}^{3} \int_{\mathbf{R}^{3}} u_{i} \partial_{i}\left(\partial_{k} u_{j} \partial_{k} u_{j}\right) d x \\
= & \sum_{i, j, k=1}^{3} \int_{\mathbf{R}^{3}} \partial_{k} u_{i} \partial_{i} u_{j} \partial_{k} u_{j} d x \\
= & \sum_{i=1}^{2} \sum_{j, k=1}^{3} \int_{\mathbf{R}^{3}} \partial_{k} u_{i} \partial_{i} u_{j} \partial_{k} u_{j} d x+\sum_{j=1}^{2} \sum_{k=1}^{3} \int_{\mathbf{R}^{3}} \partial_{k} u_{3} \partial_{3} u_{j} \partial_{k} u_{j} d x \\
& +\sum_{k=1}^{3} \int_{\mathbf{R}^{3}} \partial_{k} u_{3} \partial_{3} u_{3} \partial_{k} u_{3} d x=\sum_{m=1}^{3} I_{m} .
\end{aligned}
$$

The estimation of the terms $I_{m}$ is now estimated one by one.

In order to estimate $I_{1}$ and $I_{2}$, employing integration by parts deduces that

$$
\begin{aligned}
& I_{1}=\sum_{i=1}^{2} \sum_{j, k=1}^{3} \int_{\mathbf{R}^{3}} u_{i} \partial_{k}\left(\partial_{i} u_{j} \partial_{k} u_{j}\right) d x \leq c \int_{\mathbf{R}^{3}}|\tilde{u}||\nabla u|\left|\nabla^{2} u\right| d x, \\
& I_{2}=\sum_{j=1}^{2} \sum_{k=1}^{3} \int_{\mathbf{R}^{3}} u_{j} \partial_{3}\left(\partial_{k} u_{3} \partial_{k} u_{j}\right) d x \leq c \int_{\mathbf{R}^{3}}|\tilde{u}||\nabla u|\left|\nabla^{2} u\right| d x .
\end{aligned}
$$


For $I_{3}$, the divergence-free condition $\partial_{3} u_{3}=-\partial_{1} u_{1}-\partial_{2} u_{2}$ and integration by parts imply

$$
\begin{aligned}
I_{3} & =\sum_{k=1}^{3} \int_{\mathbf{R}^{3}} \partial_{k} u_{3}\left(\partial_{1} u_{1}+\partial_{2} u_{2}\right) \partial_{k} u_{3} d x \\
& \leq-\sum_{k=1}^{3} \int_{\mathbf{R}^{3}}\left(u_{1} \partial_{1}\left(\partial_{k} u_{3} \partial_{k} u_{3}\right)+u_{2} \partial_{2}\left(\partial_{k} u_{3} \partial_{k} u_{3}\right)\right) d x \\
& \leq c \int_{\mathbf{R}^{3}}|\tilde{u}||\nabla u|\left|\nabla^{2} u\right| d x .
\end{aligned}
$$

Thus, plugging the above inequalities into (2.8) to produce

$$
\frac{d}{d t}\|\nabla u(t)\|_{L_{2}}^{2}+2\|\Delta u(t)\|_{L_{2}}^{2} \leq c \int_{\mathrm{R}^{3}}|\tilde{u} \| \nabla u|\left|\nabla^{2} u\right| d x:=\text { RHS. }
$$

We now carry out the estimation of (2.12) based on the assumption described by (1.11). Applying Hölder's inequality and Young's-inequality, we have for the right-hand side (RHS) of (2.12)

$$
\begin{aligned}
\text { RHS } & \leq c\left\|\left|\tilde{u}\left\|\nabla u\left|\left\|_{L_{2}}\right\| \nabla^{2} u\left\|_{L_{2}} \leq c(\varepsilon)\right\|\right| \tilde{u}\right\| \nabla u\right|\right\|_{L_{2}}^{2}+\varepsilon\left\|\nabla^{2} u\right\|_{L_{2}}^{2} \\
& \leq c\|\tilde{u}\|_{L_{p, s}}^{2}\|\nabla u\|_{L_{2 p /(p-2), 2}}^{2}+\frac{1}{2}\|\Delta u\|_{L_{2}}^{2}
\end{aligned}
$$

where we have used the following Hölder inequality's in Lorentz space in the last line (refer to O'Neil [21, Theorems 3.4 and 3.5])

$$
\|f g\|_{L_{p_{1}, q_{1}}} \leq c\|f\|_{L_{p_{2}, q_{2}}}\|g\|_{L_{p_{3}, q_{3}}}
$$

for

$$
\frac{1}{p_{1}}=\frac{1}{p_{2}}+\frac{1}{p_{3}}, \quad \frac{1}{q_{1}} \leq \frac{1}{q_{2}}+\frac{1}{q_{3}}
$$

with

$$
1 \leq p_{2}, \quad p_{3} \leq \infty, \quad 1 \leq q_{2}, \quad q_{3} \leq \infty .
$$

We now claim that the term $\|\nabla u\|_{L_{2 p /(p-2), 2}}$ in (2.13) can be estimated by applying the following Gagliardo-Nirenberg inequality in Lorentz space

$$
\|\nabla f\|_{L_{2 p /(p-2), 2}} \leq c\|\nabla f\|_{L_{2}}^{(p-3) / p}\|\Delta f\|_{L_{2}}^{3 / p}
$$


Indeed, choosing $p_{1}$ and $p_{2}$ such that

$$
3<p_{1}<p<p_{2}<\infty, \quad \frac{2}{p}=\frac{1}{p_{1}}+\frac{1}{p_{2}},
$$

and then applying Gagliardo-Nirenberg inequality, it follows that

$$
\|\nabla f\|_{2 p_{i} /\left(p_{i}-2\right)} \leq c\|\nabla f\|_{L_{2}}^{\left(p_{i}-3\right) / p_{i}}\|\Delta f\|_{L_{2}}^{3 / p_{i}}, \quad i=1,2 .
$$

Thus, applying the interpolation inequality (2.3), we have

$$
L_{2 p /(p-2), 2}\left(\mathbf{R}^{3}\right)=\left(L_{2 p_{1} /\left(p_{1}-2\right)}\left(\mathbf{R}^{3}\right), L_{2 p_{2} /\left(p_{2}-2\right)}\left(\mathbf{R}^{3}\right)\right)_{1 / 2,2^{\prime}}
$$

that is to say,

$$
\begin{aligned}
\|\nabla f\|_{L_{2 p /(p-2), 2}} & \leq c\|\nabla f\|_{L_{2 p_{1} /\left(p_{1}-2\right)}}^{1 / 2}\|\nabla f\|_{L_{2 p_{2} /\left(p_{2}-2\right)}}^{1 / 2} \\
& \leq c\left(\|\nabla f\|_{L_{2}}^{p_{1}-3 / p_{1}}\|\Delta f\|_{L_{2}}^{3 / p_{2}}\right)^{1 / 2}\left(\|\nabla f\|_{L_{2}}^{\left(p_{2}-3\right) / p_{2}}\|\Delta f\|_{L_{2}}^{3 / p_{2}}\right)^{1 / 2} \\
& \leq c\|\nabla f\|_{L_{2}}^{(p-3) / p}\|\Delta f\|_{L_{2}}^{3 / p},
\end{aligned}
$$

and (2.17) is derived. Therefore, by employing (2.17) and Young's inequality, the inequality (2.13) becomes

$$
\begin{aligned}
\text { RHS } & \leq c\|\tilde{u}\|_{L_{p, \infty}}^{2}\|\nabla u\|_{L_{2}}^{2(p-3) / p}\|\Delta u\|_{L_{2}}^{6 / p}+\frac{1}{2}\|\Delta u\|_{L_{2}}^{2} \\
& \leq c\|\tilde{u}\|_{L_{p, \infty}}^{2 p /(p-3)}\|\nabla u\|_{L_{2}}^{2}+\|\Delta u\|_{L_{2}}^{2} .
\end{aligned}
$$

Inserting (2.22) into (2.12) to produce

$$
\frac{d}{d t}\|\nabla u(t)\|_{L_{2}}^{2}+\|\Delta u(t)\|_{L_{2}}^{2} \leq c\|\tilde{u}\|_{L_{p, \infty}}^{2 p /(p-3)}\|\nabla u\|_{L_{2}}^{2} .
$$

Taking Gronwall's inequality into account yields the desired estimate,

$$
\sup _{0<t<T}\|\nabla u(t)\|_{L_{2}}^{2}+\int_{0}^{T}\|\Delta u(t)\|_{L_{2}}^{2} d t \leq c\left\|\nabla u_{0}\right\|_{L_{2}}^{2} \exp \left\{\int_{0}^{T}\|\tilde{u}(t)\|_{L_{p, \infty}}^{q} d t\right\},
$$

note that

$$
\frac{2 p}{p-3}=q
$$

This completes the proof of Theorem 2.1. 


\section{Proof of Theorem 1.2}

According to a priori estimates of smooth solutions described in Theorem 2.1, the proofs of Theorem 1.2 are standard.

Since $u_{0} \in H^{1}\left(\mathbf{R}^{3}\right)$ with $\nabla \cdot u_{0}=0$, by the local existence theorem of strong solutions to the Navier-Stokes equations (see, e.g., Fujita and Kato [22]), there exist a $T^{*}>0$ and a smooth solution $\bar{u}$ of (1.1) satisfying

$$
\bar{u} \in C\left(\left[0, T^{*}\right) ; H^{1}\right) \cap C^{1}\left(\left(0, T^{*}\right) ; H^{1}\right) \cap C\left(\left[0, T^{*}\right) ; H^{3}\right), \quad \bar{u}(x, 0)=u_{0} .
$$

Note that the Leray weak solution satisfies the energy inequality (1.10). It follows from Serrin's weak-strong uniqueness criterion [2] that

$$
\bar{u} \equiv u \quad \text { on }\left[0, T^{*}\right)
$$

Thus, it is sufficient to show that

$$
T^{*}=T \text {. }
$$

Suppose that $T^{*}<T$. Without loss of generality, we may assume that $T^{*}$ is the maximal existence time for $\bar{u}$. Since $\bar{u} \equiv u$ on $\left[0, T^{*}\right)$ and by the assumptions (1.11), it follows from $a$ priori estimate (2.7) that the existence time of $\bar{u}$ can be extended after $t=T^{*}$ which contradicts with the maximality of $t=T^{*}$.

Thus, we complete the proof of Theorem 1.2.

\section{Acknowledgments}

This work is partially supported by NNSF of China (11071185) and NSF of Tianjin (09JCYBJC01800).

\section{References}

[1] J. Leray, "Sur le mouvement d'un liquide visqueux emplissant l'espace," Acta Mathematica, vol. 63, no. 1, pp. 193-248, 1934.

[2] J. Serrin, "On the interior regularity of weak solutions of the Navier-Stokes equations," Archive for Rational Mechanics and Analysis, vol. 9, pp. 187-195, 1962.

[3] Z. M. Chen and Z. Xin, "Homogeneity criterion for the Navier-Stokes equations in the whole spaces," Journal of Mathematical Fluid Mechanics, vol. 3, no. 2, pp. 152-182, 2001.

[4] Q. Chen and Z. Zhang, "Space-time estimates in the Besov spaces and the Navier-Stokes equations," Methods and Applications of Analysis, vol. 13, no. 1, pp. 107-122, 2006.

[5] Z.-M. Chen and W. G. Price, "Blow-up rate estimates for weak solutions of the Navier-Stokes equations," Proceedings of the Royal Society A, vol. 457, no. 2015, pp. 2625-2642, 2001.

[6] Z.-M. Chen and W. G. Price, "Morrey space techniques applied to the interior regularity problem of the Navier-Stokes equations," Nonlinearity, vol. 14, no. 6, pp. 1453-1472, 2001.

[7] B. Dong, G. Sadek, and Z. Chen, "On the regularity criteria of the 3D Navier-Stokes equations in critical spaces," Acta Mathematica Scientia Series B, vol. 31, no. 2, pp. 591-600, 2011.

[8] B.-Q. Dong and Z.-M. Chen, "Regularity criterion for weak solutions to the 3D Navier-Stokes equations via two velocity components," Journal of Mathematical Analysis and Applications, vol. 338, no. 1, pp. 1-10, 2008. 
[9] Y. Zhou, "A new regularity criterion for weak solutions to the Navier-Stokes equations," Journal de Mathématiques Pures et Appliquées, vol. 84, no. 11, pp. 1496-1514, 2005.

[10] P. Penel and M. Pokorný, "Some new regularity criteria for the Navier-Stokes equations containing gradient of the velocity," Applications of Mathematics, vol. 49, no. 5, pp. 483-493, 2004.

[11] I. Kukavica and M. Ziane, "One component regularity for the Navier-Stokes equations," Nonlinearity, vol. 19, no. 2, pp. 453-469, 2006.

[12] C. Cao and E. S. Titi, "Regularity criteria for the three-dimensional Navier-Stokes equations," Indiana University Mathematics Journal, vol. 57, no. 6, pp. 2643-2661, 2008.

[13] B.-Q. Dong and Z.-M. Chen, "Regularity criteria of weak solutions to the three-dimensional micropolar flows," Journal of Mathematical Physics, vol. 50, no. 10, Article ID 103525, 2009.

[14] B.-Q. Dong, Y. Jia, and Z.-M. Chen, "Pressure regularity criteria of the three-dimensional micropolar fluid flows," Mathematical Methods in the Applied Sciences, vol. 34, no. 5, pp. 595-606, 2011.

[15] B.-Q. Dong and W. Zhang, "On the regularity criterion for three-dimensional micropolar fluid flows in Besov spaces," Nonlinear Analysis: Theory, Methods E Applications, vol. 73, no. 7, pp. 2334-2341, 2010.

[16] R. Temam, Navier-Stokes Equations, Theory and Numerical Analysis, North-Holland, Amsterdam, The Netherlands, 1977.

[17] H. Kozono and N. Yatsu, "Extension criterion via two-components of vorticity on strong solutions to the 3D Navier-Stokes equations," Mathematische Zeitschrift, vol. 246, no. 1-2, pp. 55-68, 2004.

[18] Z. Zhifei and C. Qionglei, "Regularity criterion via two components of vorticity on weak solutions to the Navier-Stokes equations in $\mathbf{R}^{3}$," Journal of Differential Equations, vol. 216, no. 2, pp. 470-481, 2005.

[19] J. Bergh and J. Löfström, Interpolation Spaces, Springer, New York, NY, USA, 1976.

[20] H. Triebel, Interpolation Theory, Function Spaces, Differential Operators, vol. 18, North-Holland, Amsterdam, The Netherlands, 1978.

[21] R. O'Neil, “Convolution operators and $L(p, q)$ spaces," Duke Mathematical Journal, vol. 30, pp. 129-142, 1963.

[22] H. Fujita and T. Kato, "On the Navier-Stokes initial value problem. I," Archive for Rational Mechanics and Analysis, vol. 16, pp. 269-315, 1964. 


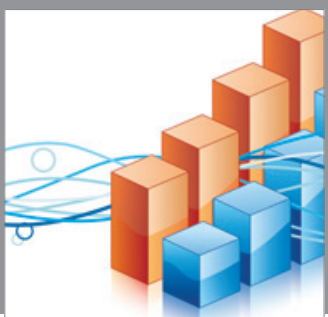

Advances in

Operations Research

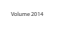

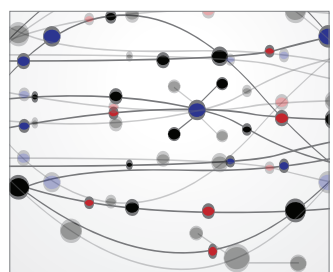

\section{The Scientific} World Journal
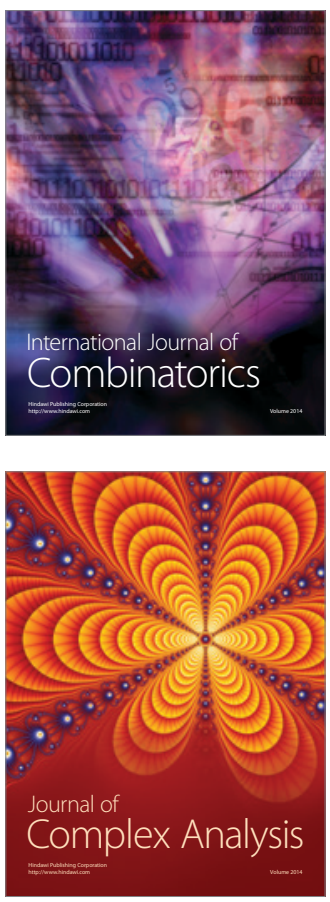

International Journal of

Mathematics and

Mathematical

Sciences
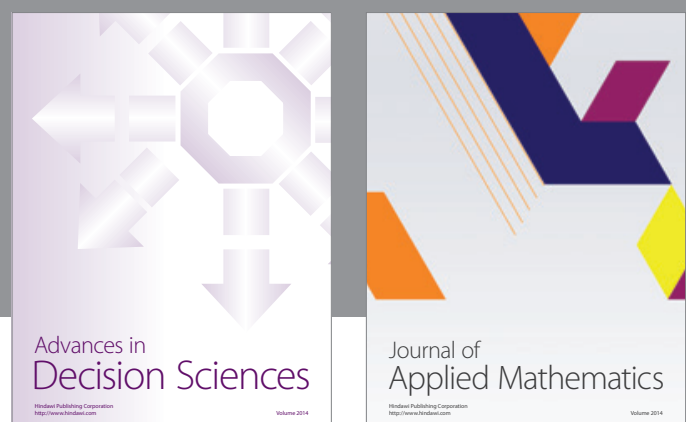

Journal of

Applied Mathematics
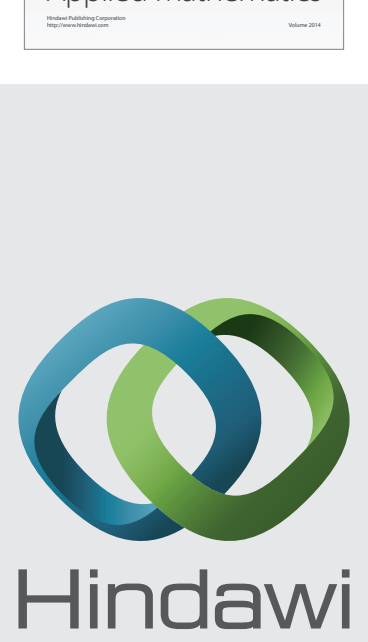

Submit your manuscripts at http://www.hindawi.com
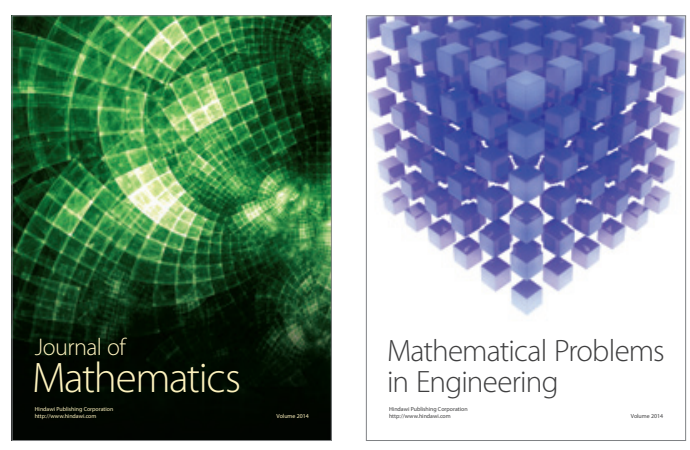

Mathematical Problems in Engineering
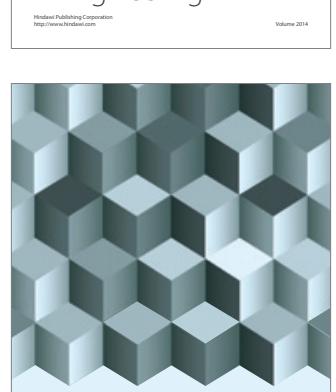

Journal of

Function Spaces
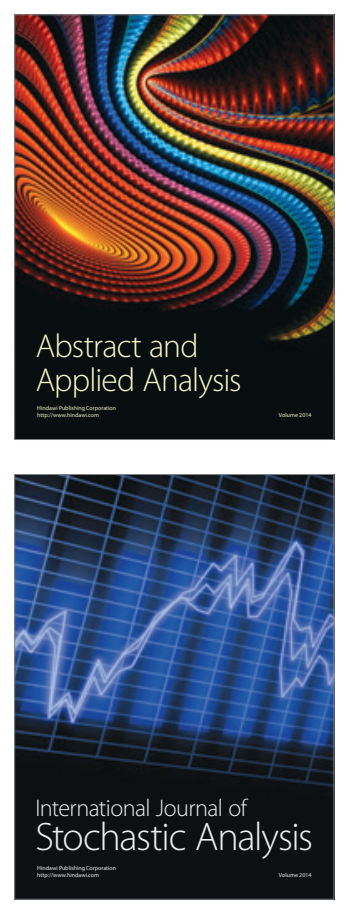

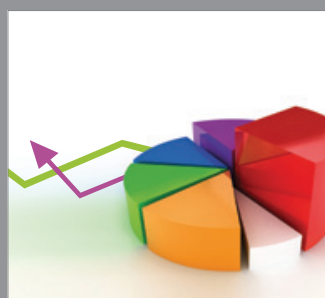

ournal of

Probability and Statistics

Promensencen
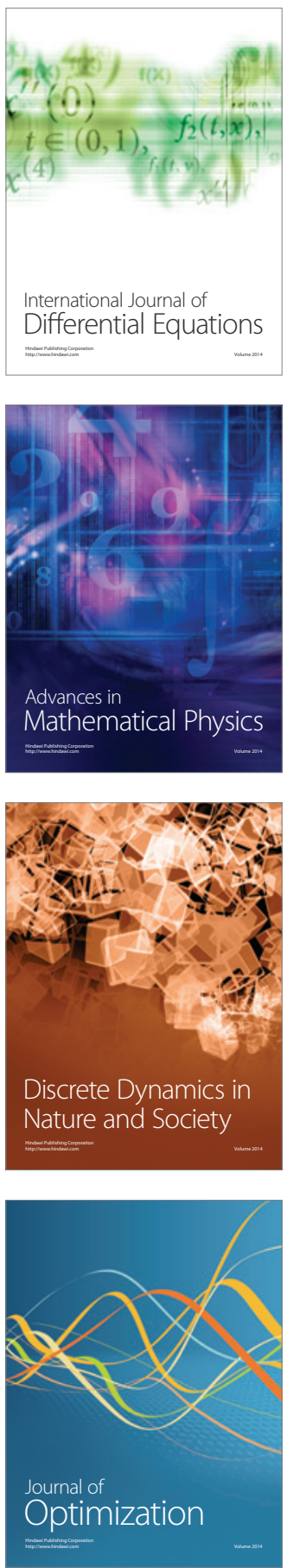